\title{
Perfil clínico e psicossocial dos moradores em hospitais psiquiátricos no estado do Ceará, Brasil
}

\author{
The clinical and psychosocial profile of inmates \\ in psychiatric hospitals in the State of Ceará, Brazil
}

Matias Carvalho Aguiar Melo ${ }^{1}$

Saulo Giovanni Castor Albuquerque ${ }^{1}$

José Henrique Sousa Luz ${ }^{1}$

Perpétua Thaís de Lima Feitosa Quental ${ }^{1}$

Alexandre Menezes Sampaio ${ }^{1}$

Alexandre Bastos Lima ${ }^{1}$

${ }^{1}$ Hospital de Saúde Mental Professor Frota Pinto. R. Vicente Nobre de Macêdo, Messejana. 60841-110 Fortaleza CE Brasil. matcarv01@yahoo.com.br

\begin{abstract}
One of the most vexing problems in the context of psychiatric reform are the inmates of psychiatric hospitals institutionalized for one year or more. The long periods of hospitalization indicate that these inmates have been abandoned, which can aggravate their psychiatric disorders. This article seeks to trace a socio-demographic and clinical profile of the inmates of psychiatric hospitals in the State of Ceará, Brazil. It is a cross-sectional study, based on reviews of medical registers, interviews and application of two scales: the Katz Index and the PANSS. Most of the 39 participants were men, single and of an economically active age. Information on education (69.2\%) and religion (66.7\%) were not known and for $12.8 \%$, their marital status was unknown. Nearly $75 \%$ received no visits from friends or relatives. Two thirds maintained total independence to perform ADLs (Activities of Daily Living). The majority were admitted for primary psychotic disorder (76.8\%). These manifested a marked negative syndrome in $96.7 \%$ of cases. The study highlights the situation of abandonment and loss of citizenship experienced by chronically institutionalized patients. It is questionable to what extent hospitals are prepared to offer the assistance that this population needs.
\end{abstract}

Key words Inmates, Psychiatric hospitals, Ceará, Brazil
Resumo Um dos mais preocupantes problemas no contexto da Reforma Psiquiátrica são os moradores dos hospitais psiquiátricos (pacientes institucionalizados por um ano ou mais). As longas internações evidenciam a situação de abandono e podem descompensar transtornos psiquiátricos. $O$ objetivo deste artigo é traçar o perfil sociodemográfico e clínico da população dos moradores de hospitais psiquiátricos do Estado do Ceará. Tratase de um estudo transversal, baseado em revisões de prontuários, entrevistas e aplicação de escalas (Índice de Katz e PANSS). Dos 39 participantes, a maioria era homem, solteiro e em idade economicamente ativa. Da maioria não se dispõe dados sobre a escolaridade $(69,2 \%)$ e a religião $(66,7 \%)$; e $12,8 \%$ têm o estado civil ignorado. Cerca de $3 / 4$ não recebiam visitas de amigos ou familiares. Dois terços mantêm independência total para a realização de AVD (Atividades de Vida Diária). A maioria estava internada por transtorno psicótico primário (76,8\%). Nesses pacientes, destacava-se a exuberância de sintomas negativos, configurando uma sindrome negativa em $96,7 \%$ dos casos. $O$ estudo destaca a situação de abandono e perda de cidadania dos pacientes cronicamente institucionalizados. Questiona-se até que ponto os hospitais estão preparados para oferecer-lhes a devida assistência.

Palavras-chave Moradores, Hospitais psiquiátricos, Ceará, Brasil 


\section{Introdução}

Segundo estimativas da Organização Mundial da Saúde (OMS), aproximadamente 450 milhões de pessoas sofrem de transtornos mentais ou comportamentais ${ }^{1}$. Esse número representa $12 \%$ da carga mundial de doenças, embora os investimentos nessa área correspondam a menos de $1 \%$ dos gastos em saúde pública. Cerca de $40 \%$ dos países sequer desenvolvem políticas de saúde mental ${ }^{2}$ e mais de $90 \%$ excluem crianças e adolescentes ${ }^{3,4}$. Diante dessa situação, apenas uma pequena minoria das pessoas acometidas por transtornos psiquiátricos recebe algum tipo de tratamento $0^{5,6}$ Inserido nesse contexto, o Brasil passa por um período de transiçãao ${ }^{7,8}$. A Reforma Psiquiátrica propõe a progressiva substituição de um modelo de cuidado em saúde mental centrado em hospitais psiquiátricos por uma rede de serviços de saúde descentralizada, buscando suporte na estruturação dos serviços em psiquiatria em três níveis de complexidade e em centros de proteção social ${ }^{9,10}$. No entanto, a forma como vem sendo implementado esse novo modelo não está livre de $\operatorname{críticas}^{11,12}$. Muito se questiona a redução de leitos em hospitais psiquiátricos sem o fortalecimento de uma rede que preste adequada assistência a esse grupo de pacientes ${ }^{13}$.

Um dos assuntos mais discutidos relacionados à Reforma Psiquiátrica diz respeito à situação dos "crônico-residentes" ou moradores dos hospitais psiquiátricos (pessoas institucionalizadas por longos períodos, seja por abandono, seja por falta de condições psicológicas ou socioeconômicas de cuidado por parte dos parentes e amigos $)^{14,15}$. Em 2005, eles ocupavam mais de 50\% dos leitos psiquiátricos públicos do Estado de São Paulo ${ }^{16}$. Dados como esse suscitam o questionamento sobre o que fazer com as pessoas em condições de alta nos hospitais psiquiátricos, mas sem suporte familiar ou de qualquer outra natureza ${ }^{17}$.

A noção de residir, morar, habitar remete ao termo "lar" como local de intimidade entre as pessoas, de sentimento de pertença e de fortes vínculos interpessoais ${ }^{18}$. Esse bem-estar é difícil de ser atingido em um local onde se convive com desconhecidos e as rotinas são impostas verticalmente e baseadas em um modelo de assistência médica que não leva em conta as singularidades.

A descompensação de transtornos psiquiátricos, manifestada através de crises de agitação e agressividade, motiva situações de abandono em internações psiquiátricas por parte da família. Este abandono pode contribuir ainda mais para o agravamento do quadro psiquiátri- co, em virtude de rotinas padronizadas que negam a subjetividade e a autonomia dos pacientes $^{19}$. Assim, pessoas internadas durante crises agudas passam a manifestar crises recorrentes favorecidas pelo próprio ambiente asilar ${ }^{20,21}$ Uma alternativa para o problema são os serviços de residências terapêuticas (SRT). As primeiras experiências surgiram no Brasil na década de 90, porém esses serviços foram incorporados à rede de atenção em saúde mental vinculada ao Sistema Único de Saúde (SUS) em $2000^{22}$. As residências terapêuticas, diferentes dos hospitais, aproximam-se do conceito de moradia, lar, uma vez que lembra uma residência convencional, está inserida na comunidade e possibilita o desenvolvimento de vínculos com vizinhos e certos grupos sociais ${ }^{17}$.

Até dezembro de 2010, o número de residências terapêuticas atingiu 753 . Desse total, 570 estavam em funcionamento, enquanto 183 permaneciam inativas ${ }^{17}$. Esse número de SRT ainda se mostra insuficiente, considerando que $30 \%$ dos leitos psiquiátricos públicos são ocupados por uma população cronificada, o que significa 9.820 potenciais moradores de residências terapêuticas. Para suprir essa demanda, seria necessária a criação de mais 1.227 novos SRT, que, somados aos 753 já existentes, totalizariam 1.980 serviços de residências terapêuticas.

No contexto da Reforma Psiquiátrica, tornase indispensável conhecer a identidade da população de residentes-crônicos de hospitais psiquiátricos, suas limitações, a gravidade de seu quadro clínico e o(s) motivo(s) da internação prolongada. A ciência desses dados ajudará a traçar a melhor forma de desinstitucionalizar esses pacientes e o destino mais adequado após a alta hospitalar ${ }^{16}$. Apesar da grande relevância do tema em termos individuais e coletivos, poucas pesquisas foram desenvolvidas com o enfoque na população de moradores de hospitais psiquiátricos ${ }^{23,24}$.

Este trabalho tem por objetivo definir as características da população de moradores de instituições hospitalares do estado do Ceará, bem como avaliar sua funcionalidade e a gravidade dos sintomas apresentados (em caso de síndromes psicóticas). Embora algumas pesquisas tenham como foco a aplicação da "Positive and Negative Syndrome Scale (PANSS)" em pacientes psicóticos crônicos e do Índice de Katz em pacientes institucionalizados ${ }^{25-28}$, não se conhecem estudos publicados no Brasil que avaliem o perfil clínico e funcional (em termos de Atividades de Vida Diária - AVD) da população cronicamente institucionalizada em hospitais psiquiátricos. 
Trata-se de um estudo transversal e descritivo que avaliou todos os pacientes institucionalizados por mais de um ano em hospitais psiquiátricos, a partir de documentos emitidos por estes em 2012. Foram convidados a participar do estudo os cinco hospitais psiquiátricos cearenses com leitos públicos que registraram pacientes institucionalizados por mais um ano. No entanto, apenas de três deles se obteve consentimento escrito: Hospital de Saúde Mental Professor Frota Pinto, Hospital Nosso Lar e Hospital Psiquiátrico São Vicente de Paulo.

Por esse motivo, foi avaliada apenas uma amostra da população de pacientes cronicamente institucionalizados, correspondente a $50 \%$ do total. Para serem incluídos no estudo, os pacientes deveriam ter idade igual ou superior a 18 anos. Como critério de exclusão foi estabelecida a recusa do paciente, instituição ou pessoa responsável em participar do estudo.

A coleta de dados foi realizada ao longo de seis meses, baseado em revisões de prontuários e entrevistas com pacientes moradores e funcionários dos hospitais. Essa tarefa foi realizada por um grupo de três médicos residentes em Psiquiatria previamente treinados para padronizar a aplicação dos questionários e escalas.

O diagnóstico foi estabelecido de acordo com a 10a Classificação Internacional de Doenças (CID-10). Foram considerados apenas os registros em prontuários. Não se tentou aplicar questionários ou entrevistas estruturadas com essa finalidade.

\section{Instrumentos}

\section{Índice de Katz}

A avaliação do grau de dependência de pacientes cronicamente institucionalizados pode ser estimada por escalas de AVD.

Um dos instrumentos mais utilizados pela alta confiabilidade e fácil aplicação é o Índice de Katz, criado em 1963 por Sidney Katz ${ }^{29}$. Ele avalia seis funções (capacidade de alimentarse, controlar as funções eliminatórias, transferir-se da cama para cadeira e vice-versa, usar o banheiro, vestir-se e banhar-se) ${ }^{29}$. Cada uma das funções é pontuada da seguinte forma: 0 (se realiza as atividades de forma independente), 1 (com ajuda não humana), 2 (com ajuda humana) e 3 (totalmente dependente). Os valores desses itens são somados, e os pacientes são classificados em independência (0-5 pontos), dependência parcial (6-12 pontos) e dependência total (13-18 pontos).

\section{PANSS}

A PANSS (Positive and Negative Syndrome Scale) é uma escala útil na avaliação dos sintomas positivos e negativos da esquizofrenia ${ }^{30}$, sendo é composta pelos 18 itens da BPRS (Brief Psychiatry Rating Scale) e por 12 adicionais da Psychopathology Rating. Se divide em 3 grupos: sintomas positivos (7 itens), sintomas negativos (7 itens) e sintomas de psicopatologia geral (16 itens). A cada sintoma, são atribuídos escores que variam em sete níveis de gravidade da psicopatologia (de 1 a 7). A presença de três ou mais sintomas positivos ou negativos, com pontuação igual ou superior a quatro, traduz, respectivamente, síndrome positiva e negativa. Se ambas coexistirem, denomina-se síndrome mista. Neste estudo, a escala foi aplicada a todos os moradores com diagnóstico de esquizofrenia ou psicose não orgânica e não especificada.

\section{Análise dos dados}

Os dados obtidos foram tabulados e analisados pelo SPSS (Statistical Package for the Social Sciences) $-17^{\text {a }}$ versão e expostos por meio de gráficos e tabelas.

Para avaliar associações estatísticas, foi empregado o Teste Qui-Quadrado no caso de duas variáveis categóricas. Convencionou-se o intervalo de confiança superior a $95 \%(\mathrm{p}<0,05)$ para considerar os resultados estatisticamente significativos. Outros testes foram usados quando variáveis não categóricas foram analisadas.

\section{Aspectos Éticos}

A presente pesquisa seguiu os princípios éticos estabelecidos pelo Conselho Nacional de Saúde (CNS) e foi aprovada no Comitê de Ética e Pesquisa da Escola de Saúde Pública do Estado do Ceará (ESP-CE). A inclusão do paciente no estudo somente ocorreu mediante a assinatura do Termo de Consentimento Livre e Esclarecido (TCLE), após uma explicação detalhada dos objetivos e procedimentos a serem realizados durante o trabalho. A participação na pesquisa foi voluntária; e o anonimato, preservado. Nos casos de comprometimento do juízo crítico da realidade, solicitou-se a concordância de representante legal, do familiar ou do responsável pela instituição que o abriga. 


\section{Resultados}

\section{Dados Sociodemográficos}

Foram contabilizados 78 pacientes internados por período igual ou superior a um ano nos 760 leitos públicos de hospitais psiquiátricos em todo o Ceará. Portanto, 10,3\% de todos os leitos públicos eram ocupados por pessoas cronicamente institucionalizadas.

Os três hospitais psiquiátricos que participaram da pesquisa registraram 41 moradores, dos quais dois já haviam recebido alta no momento da entrevista. Portanto, 39 pacientes participaram da pesquisa, valor equivalente à metade da população de moradores de hospitais psiquiátricos do Ceará na mesma época.

A Tabela 1 apresenta o perfil sociodemográfico dessa população. A maioria era do gênero masculino $(64,1 \%)$ e procedente da capital $(87,2 \%)$. O estado civil de cinco moradores era ignorado. Os demais eram todos solteiros. Em relação à faixa etária, a maior

Tabela 1. Perfil sociodemográfico dos moradores de hospitais psiquiátricos cearenses.

\begin{tabular}{|c|c|c|}
\hline & $\mathbf{N}$ & $\%$ \\
\hline \multicolumn{3}{|l|}{ Gênero } \\
\hline Masculino & 25 & 64,1 \\
\hline Feminino & 14 & 35,9 \\
\hline \multicolumn{3}{|l|}{ Faixa Etária } \\
\hline 20 a 39 anos & 12 & 30,8 \\
\hline 40 a 59 anos & 20 & 51,2 \\
\hline 60 anos ou mais & 7 & 18 \\
\hline \multicolumn{3}{|l|}{ Estado Civil } \\
\hline Solteiro & 34 & 87,2 \\
\hline Casado, Viúvo ou Divorciado & 0 & \\
\hline Ignorado & 5 & 12,8 \\
\hline \multicolumn{3}{|l|}{ Religião } \\
\hline Católica & 9 & 23 \\
\hline Evangélica & 1 & 2,6 \\
\hline Sem Religião & 3 & 7,7 \\
\hline Ignorada & 26 & 66,7 \\
\hline \multicolumn{3}{|l|}{ Procedência } \\
\hline Capital & 34 & 87,2 \\
\hline Interior & 3 & 7,7 \\
\hline Ignorado & 2 & 5,1 \\
\hline \multicolumn{3}{|l|}{ Escolaridade } \\
\hline Analfabeto & 5 & 12,8 \\
\hline 1 a 7 anos de estudo & 4 & 10,3 \\
\hline 8 a 12 anos de estudo & 2 & 5,1 \\
\hline Ensino Superior Incompleto & 1 & 2,6 \\
\hline Ignorado & 27 & 69,2 \\
\hline
\end{tabular}

parte dos moradores se concentrava no grupo da meia-idade (40 a 59 anos). Havia sete idosos; um deles com idade superior a 80 anos. A religião e a escolaridade da maioria dos moradores eram ignoradas. Os demais são predominantemente católicos e com baixo grau de escolaridade.

\section{Dados Clínicos}

A Figura 1 relaciona os diagnósticos da população de moradores de hospitais psiquiátricos. A maior parte estava internada por um transtorno psicótico primário $(76,8 \%)$. Destaca-se, contudo, o grande número de pacientes que necessitam ter seu diagnóstico melhor investigado $(35,8 \%)$, tendo sido descritos como psicoses orgânicas não especificadas ou esquizofrenias outras ou não especificadas.

Em relação a incapacidades, 15,4\% apresentam algum tipo de deficiência grave, não corrigida e reconhecida pela equipe de saúde, seja visual $(7,7 \%)$, auditiva $(2,6 \%)$ ou motora $(5,1 \%)$, sendo os últimos limitados ao leito.

A Figura 2 exibe informações sobre o tempo de internação em hospitais psiquiátricos. Observa-se que $48,7 \%$ estavam institucionalizados por um período superior a três anos; e 35,9\%, por mais de cinco anos. Quanto à situação jurídica das internações dos moradores, a maioria delas foi voluntária (59\%); e 5,1\%, compulsórias.

A Tabela 2 se refere ao perfil nutricional dos moradores dos hospitais psiquiátricos. Quase metade dos pacientes $(48,7 \%)$ avaliados não estão na faixa adequada de IMC $(18,5-24,9)$. Uma parcela importante $(7,7 \%)$ apresenta IMC inferior a 16 , configurando um quadro de desnutrição grave ou grau 3.

\section{Vínculos Sociais}

Em relação à interação social, 74,4\% dos moradores não recebem visitas; e $69,2 \%$ não recebem qualquer benefício ou ajuda da família para as despesas.

Considerando a capacidade de deslocamento, a maioria $(74,4 \%)$ transita pelo hospital desacompanhado e sem assistência. Sobre a participação dos moradores em atividades de entretenimento e integração desenvolvidas no próprio hospital, a maioria (41\%) não participa; e apenas $12,8 \%$ participam espontaneamente e sem necessidade de auxílio.

Quanto às habilidades de comunicação, um terço $(33,3 \%)$ não consegue estabelecer 


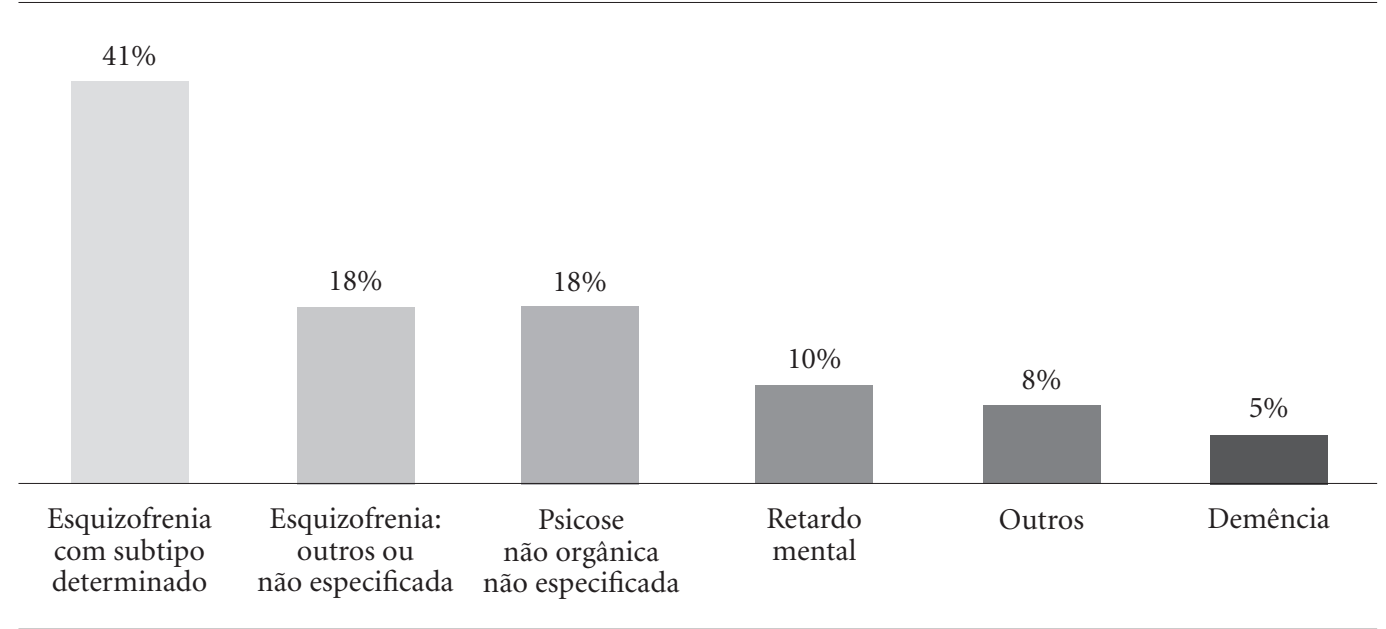

Figura 1. Diagnósticos dos moradores de hospitais psiquiátricos cearenses.

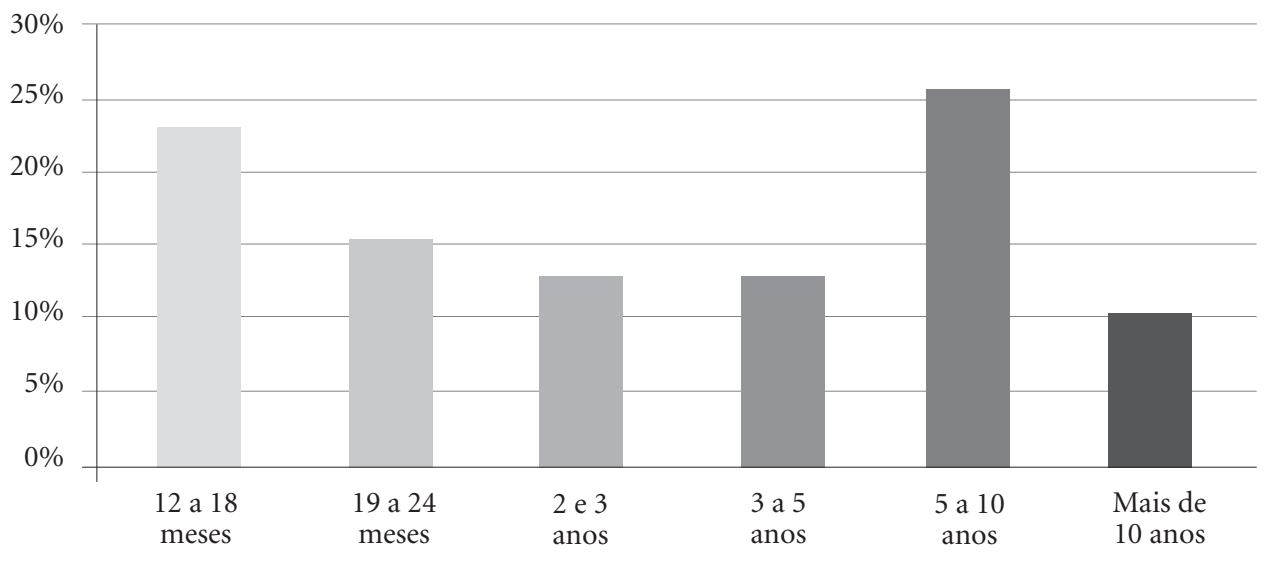

Figura 2. Tempo de internação dos moradores de hospitais psiquiátricos cearenses.

Tabela 2. Perfil nutricional dos moradores de hospitais psiquiátricos cearenses.

\begin{tabular}{lrrr}
\hline & \multicolumn{1}{c}{ IMC } & N & \% \\
\hline Eutróficos & $18,5-24,9$ & 20 & 51,3 \\
Desnutrição Leve (Grau 1) & $17-18,49$ & 4 & 10,2 \\
Desnutrição Moderada (Grau 2) & $16-16,9$ & 1 & 2,6 \\
Desnutrição Grave (Grau 3) & $<16$ & 3 & 7,7 \\
Sobrepeso & $24,9-29,9$ & 9 & 23 \\
Obesidade Grau 1 & $30-34,9$ & 1 & 2,6 \\
Obesidade Grau 2 & $35-39,9$ & 1 & 2,6 \\
Obesidade Grau 3 & $>40$ & 0 & 0 \\
\hline
\end{tabular}

uma comunicação eficaz (nem quando solicitados ou auxiliados). Cerca de 35,9\% apresentam importante dificuldade de comunicação, que ocorre apenas esporadicamente ou quando solicitados ou auxiliados por terceiros. Comunicam-se espontânea e regularmente, 30,8\%.

\section{Atividades de Vida Diária}

Avaliando as atividades de vida diária (AVD), a pontuação média do Índice de Katz foi de 5,15 . Dois terços dos moradores são considerados independentes para as AVD; e apenas 12,8\% são totalmente dependentes, de acordo com o Índice de Katz. O banho e a troca de roupa fo- 
ram as atividades de que os moradores precisaram de mais ajuda; e o deslocamento e a alimentação foram as mais realizadas sem auxílio.

\section{Avaliação dos sintomas psicóticos}

Dentre os pacientes avaliados no estudo, $76,8 \%$ deles receberam o diagnóstico de esquizofrenia ou psicose não orgânica e não especificada. A estes foi aplicada a escala PANSS com a finalidade de avaliar sintomas negativos e positivos. Os resultados encontram-se resumidamente descritos na Tabela 3.

A média de pontuação dos sintomas positivos e negativos foi, respectivamente, 19,3 e 35. A maioria dos pacientes manifestava uma síndrome negativa pura $(63,4 \%)$. Apresentavam síndrome mista, $33,3 \%$; e uma pessoa $(3,3 \%)$ não tinha síndrome. Dos sintomas positivos, o que mais se destaca é a desorganização conceitual. Quanto aos sintomas negativos, cada um deles estava presente de forma relevante em mais de dois terços dos participantes.

Evidenciou-se uma correlação significativamente estatística positiva da pontuação de sintomas negativos na PANNS com o Índice de Katz ( $\mathrm{p}=0,002)$ e com a ausência de uma comunicação eficaz ( $\mathrm{p}<0,001)$. Portanto, quanto maior a pontuação para sintomas negativos na PANSS entre os moradores, maior a pontuação no Índice de Katz (grau de dependência de AVD) e maior a incapacidade para comunicação.

Não houve associações estatisticamente significativas entre pontuação de sintomas negativos na PANNS e idade igual ou superior a 60 anos, tempo de internação, participação de atividades no hospital, estado nutricional e presença de deficiências físicas.

\section{Discussão}

Embora existam outras pesquisas sobre pacientes cronicamente institucionalizados em hospitais psiquiátricos $^{16,31,32}$, este estudo parece ter sido o primeiro do Brasil a objetivamente avaliar, mediante índices e escalas, seu estado nutricional, seu grau de dependência funcional e sua sintomatologia positiva e negativa (em caso de esquizofrenia e transtorno psicótico não orgânico e não especificado).

Os pacientes cronicamente institucionalizados ocupavam 10,3\% dos leitos públicos dos hospitais psiquiátricos do Ceará, valor pequeno se comparado a outras épocas e locais ${ }^{14,33-35}$.
Tabela 3. Sintomas positivos e negativos dos moradores psicóticos primários de hospitais psiquiátricos cearenses.

\begin{tabular}{|c|c|c|}
\hline & $\mathrm{N}$ & $\%$ \\
\hline \multicolumn{3}{|c|}{ Tipo de Síndrome } \\
\hline Positiva & 0 & 0 \\
\hline Negativa & 19 & 63,4 \\
\hline Mista & 10 & 33,3 \\
\hline Ausente & 1 & 3,3 \\
\hline \multicolumn{3}{|c|}{ Sintomas Positivos } \\
\hline \multicolumn{3}{|c|}{ Delírios } \\
\hline Escore $<4$ & 22 & 73,3 \\
\hline Escore $\geq 4$ & 8 & 26,7 \\
\hline \multicolumn{3}{|c|}{ Desorganização Conceitual } \\
\hline Escore $<4$ & 4 & 13,3 \\
\hline Escore $\geq 4$ & 26 & 86,7 \\
\hline \multicolumn{3}{|c|}{ Comportamento Alucinatório } \\
\hline Escore $<4$ & 20 & 66,7 \\
\hline Escore $\geq 4$ & 10 & 33,3 \\
\hline \multicolumn{3}{|l|}{ Excitação } \\
\hline Escore $<4$ & 23 & 76,7 \\
\hline Escore $\geq 4$ & 7 & 23,3 \\
\hline \multicolumn{3}{|l|}{ Grandeza } \\
\hline Escore $<4$ & 29 & 96,7 \\
\hline Escore $\geq 4$ & 1 & 3,3 \\
\hline \multicolumn{3}{|l|}{ Desconfiança } \\
\hline Escore $<4$ & 21 & 70 \\
\hline Escore $\geq 4$ & 9 & 30 \\
\hline \multicolumn{3}{|l|}{ Hostilidade } \\
\hline Escore $<4$ & 26 & 86,7 \\
\hline Escore $\geq 4$ & 4 & 13,3 \\
\hline \multicolumn{3}{|c|}{ Sintomas Negativos } \\
\hline \multicolumn{3}{|c|}{ Empobrecimento Afetivo } \\
\hline Escore $<4$ & 9 & 30 \\
\hline Escore $\geq 4$ & 21 & 70 \\
\hline \multicolumn{3}{|c|}{ Retraimento Emocional } \\
\hline Escore $<4$ & 4 & 13,3 \\
\hline Escore $\geq 4$ & 26 & 86,7 \\
\hline \multicolumn{3}{|l|}{ Contato Pobre } \\
\hline Escore $<4$ & 9 & 30 \\
\hline Escore $\geq 4$ & 21 & 70 \\
\hline \multicolumn{3}{|c|}{ Retraimento Social } \\
\hline Escore $<4$ & 3 & 10 \\
\hline Escore $\geq 4$ & 27 & 90 \\
\hline \multicolumn{3}{|c|}{ Dificuldade no Pensamento Abstrato } \\
\hline Escore $<4$ & 0 & 0 \\
\hline Escore $\geq 4$ & 30 & 100 \\
\hline \multicolumn{3}{|c|}{ Falta de Espontaneidade e Fluência } \\
\hline \multicolumn{3}{|c|}{ Verbal } \\
\hline Escore $<4$ & 4 & 13,3 \\
\hline Escore $\geq 4$ & 26 & 86,7 \\
\hline \multicolumn{3}{|c|}{ Pensamento Estereotipado } \\
\hline Escore $<4$ & 4 & 13,3 \\
\hline Escore $\geq 4$ & 26 & 86,7 \\
\hline
\end{tabular}


O estudo destaca a situação de abandono. Apenas 25,6\% desses pacientes recebiam visitas de amigos e familiares; e 69,2\% não recebem qualquer tipo de renda, dificultando um processo futuro de integração social. Essa situação de abandono também é registrada por outros trabalhos brasileiros que abordam a mesma temática, mas parece menos crítica ${ }^{16,31}$. Em um censo realizado em 2008 no Estado de São Paulo, 28\% dos moradores de hospitais psiquiátricos não recebem visitas de amigos ou familiares. Em outra pesquisa realizada em Natal e publicada em 2009, esse número foi de 55\%. Ambos os valores são menores do que os do presente estudo. Cerca de $35 \%$ da população institucionalizada natalense e $52,8 \%$ da paulista não recebem qualquer benefício, percentuais maiores que os do Ceará.

A perda da identidade também parece mais marcante na realidade cearense. Enquanto que o estado civil e a religião eram ignorados respectivamente em 7,2\% e 36\% em São Paulo ${ }^{16}$, esses valores são aumentados para 12,8\% e 66,7\% em nosso estado. Na nossa amostra, a maioria não dispunha de informações básicas, como escolaridade $(69,2 \%)$; e de $5,1 \%$ não se sabia a procedência. Mesmo os diagnósticos necessitavam de melhor esclarecimento em pelo menos 35,9\% dos casos. Isso reforça o processo de marginalização da sociedade e descaracterização de identidade a que essas pessoas estão submetidas.

Essa situação de abandono e perda da identidade se torna mais instigante ao se verificar que a maioria dos moradores (82\%) tinha entre $20 \mathrm{e}$ 59 anos (idade economicamente ativa), ou seja, tinha idade potencial para exercício de atividades laborais. No entanto, esse valor é similar ao encontrado nas internações psiquiátricas em geral na cidade $(82,6 \%)^{36}$.

Quanto à procedência, merece atenção a grande proporção de pacientes procedentes da capital $(87,2 \%)$. Apenas $7,7 \%$ vieram do interior e de 5,1\% se ignorava essa informação. Essa discrepância entre a quantidade de moradores da capital e do interior supera a que já costuma ocorrer em internações em geral. Outro estudo também realizado em Fortaleza ${ }^{36}$, por exemplo, apontou que $17,55 \%$ de todos os pacientes internados voluntária e involuntariamente em 2007 provieram do interior, valor bem maior que o encontrado dentre os moradores. Esses dados contrariam a hipótese de um possível predomínio de moradores dentre pacientes do interior, cogitando uma possível relação entre o abandono e a distância da cidade de origem.
Dessa forma, fazem-se necessários mais estudos com a finalidade de explicar esse achado. Considerando o tempo de internação, é instigante a constatação de que $48,7 \%$ estavam institucionalizados por um período superior a três anos; e 35,9\%, por mais de cinco anos. Segundo outros estudos, o longo tempo de internação pode piorar as manifestações clínicas das doenças psiquiátricas ${ }^{17,18,22}$.

Em relação aos aspectos jurídicos das internações, 59\% dos moradores de hospitais psiquiátricos foram voluntariamente internados. Esse dado induz o questionamento se os pacientes realmente concordaram com as próprias internações que resultaram em uma situação de abandono para a maioria, se eles tinham capacidade (à época das internações) de autodeterminação ou se essa classificação foi erroneamente empregada. Além disso, 5,1\% das internações são compulsórias, tema que exige maior reflexão sobre os motivos de manter um paciente internado por tanto tempo por ordem judicial.

Quase metade dessas pessoas não apresenta um estado nutricional satisfatório, de acordo com cálculos do IMC (Índice de Massa Corpórea), mesmo residindo em um hospital, local de referência em cuidados de saúde. Dos pacientes, 20,5\% foram diagnosticados com desnutrição e 7,7\% com desnutrição grave. Esse número de desnutridos é cerca de quatro vezes superior à prevalência de desnutrição entre adultos acompanhados na assistência básica em 2007 no Ceará $(4,9 \%)$, de acordo com o DATASUS, departamento de informática do SUS ${ }^{37}$. Já em relação a sobrepeso e obesidade, a prevalência na população estudada foi de $23 \%$ e 5,1\%, respectivamente, valores inferiores aos de adultos em 2007 no Ceará (30,5\% e 13,3\%, respectivamente). Esses dados levam à reflexão sobre o quanto a doença de base ou a carência de uma assistência interdisciplinar apropriada estariam contribuindo para menores valores de IMC nos pacientes cronicamente institucionalizados. Para isso, urge a realização de estudos que considerem a influência de diversas variáveis nesse processo.

É significativo o número de deficiências físicas importantes e não corrigidas $(15,4 \%)$ e de limitação ao próprio leito $(5,1 \%)$ dentre os moradores de hospitais psiquiátricos. Isso suscita questionamentos sobre a adequação da estrutura física dos hospitais psiquiátricos e a disponibilidade de um número suficiente de profissionais para atender às demandas dessas pessoas. Constata-se, dentre os moradores, um reduzido 
repertório de habilidades interpessoais: $64,1 \%$ deles têm a capacidade de comunicação bastante dificultada ou até impossibilitada; e 87,2\% não participam das atividades de integração e entretenimento desenvolvidas no hospital ou necessitam de terceiros para realizá-las. Isso acentua seu estado de isolamento e remete a um provável benefício da residência em comunidades terapêuticas, que podem oferecer uma inserção social real.

Dois terços dos moradores são considerados independentes para a execução de AVD, ou seja, conseguem garantir seus autocuidados de maneira satisfatória. Assim, quanto à funcionalidade, não se justifica sua permanência em uma instituição hospitalar. Eles parecem aptos a continuar seu tratamento numa residência terapêutica.

Em relação aos aspectos clínicos, a maioria dos pacientes do estudo estava internada por transtorno psicótico primário (76,8\%). Nesses pacientes, destaca-se a exuberância de sintomas negativos, configurando uma síndrome negativa em $96,7 \%$ dos casos: $63,4 \%$ na forma pura e $33,3 \%$ associados à importante sintomatologia positiva (síndrome mista). Portanto, a maior parte dos moradores manifesta uma síndrome negativa pura.

Questiona-se se sintomas negativos são suficientes para justificar uma internação psiquiátrica, uma vez que poucos respondem às medicações e não parecem resultar em um risco a si e a terceiros. Internações prolongadas podem até contribuir para agravá-los, uma vez que limitam a autonomia dos pacientes e dificultam sua socialização.

A maior pontuação de sintomas negativos na PANSS correlacionou-se de forma estatisticamente significativa com maiores escores no Índice de Katz (maior dependência) e menor repertório de comunicação. Dessa forma, uma piora clínica está diretamente relacionada a uma menor funcionalidade.

Poder-se-ia cogitar associações com outras variáveis (como participação em atividades e tempo de internação), mas nenhuma delas foi estatisticamente significativa. No entanto, o pequeno tamanho da população de moradores avaliada pode ter contribuído para a falta de significância estatística.

\section{Limitações}

Uma limitação importante do estudo foi o fato de se ter avaliado uma parte (e não a totalidade) dos pacientes cronicamente institucionalizados. A não participação de dois hospitais limita a extensão de seus resultados. No entanto, foi avaliada metade da população, o que torna a amostra representativa do estado.

O preenchimento indevido dos prontuários (lacunas de informações ou letras ilegíveis) pode ter comprometido parcialmente a pesquisa.

Questiona-se também se os profissionais entrevistados (assistentes sociais e auxiliares de enfermagem de plantão nos respectivos hospitais) foram as pessoas mais adequadas para fornecer dados sobre os pacientes. Muitas das informações que eles desconheciam e que não constavam no prontuário foram aventadas como ignoradas.

Além disso, adotou-se apenas o diagnóstico que era informado no prontuário, passível de erros. O emprego de escalas e questionários estruturados para avaliação diagnóstica poderia otimizar essa avaliação. A aplicação de testes neuropsicológicos também poderia melhor avaliar a dimensão cognitiva dos pacientes psicóticos e identificar as funções mentais mais comprometidas.

\section{Conclusão}

O estudo expõe a situação de abandono e perda de identidade que os pacientes cronicamente institucionalizados vivenciam, o que se constata a partir do grande número de pessoas que não recebem visitas de amigos ou familiares e têm informações básicas (como relacionadas à religião e escolaridade) ignoradas.

A maior parte dessas pessoas mostra-se independente para execução dos autocuidados e persiste apenas com sintomas negativos da doença (nos casos dos psicóticos), levando ao questionamento se o internamento se faz necessário ou se pode estar agravando a sintomatologia clínica e contribuindo para uma piora funcional.

Merece destaque a relação estatisticamente significativa entre sintomas negativos da esquizofrenia e prejuízo na realização de AVD, mostrando o quanto a clínica e a funcionalidade dos pacientes estão intrinsecamente associadas.

\section{Colaboradores}

MCA Melo, SGC Albuquerque, JHS Luz, PTLF Quental, AM Sampaio e AB Lima participaram igualmente de todas as etapas de elaboração do artigo. 


\section{Referências}

1. Mangia EF, Rosa CA. Desinstitucionalização e serviços residenciais terapêuticos. Rev Ter Ocup 2002; 13(2):7177.

2. Lund C. Quais os princípios que devem nortear as políticas de saúde mental em países de baixa e média rendas. Rev Bras Psiquiat 2010; 32(4):349-350.

3. Belfer Ml, Saxena S. WHO Child Atlas project. Lancet 2006; 367(9510):551-552.

4. Patel V, Flisher AJ, Hetrick S, McGorry P. Mental health of young people: a global public-health challenge. Lancet 2007; 369(9569):1302-1313.

5. Associação Brasileira de Psiquiatria. Diretrizes para um modelo de assistência integral em saúde mental no Brasil. [S.l: s.n.], 2006 [acessado 2013 fev 13]. Disponível em: http://www.abp.org.br/diretrizes_final.pdf

6. Roy R, Jahan M, Kumari S, Chakraborty PK. Reasons for Drug Non-Compliance of Psychiatric Patients: A Centre Based Study. JIAAP 2005; 31(1):24-28.

7. Hirdes A. A reforma psiquiátrica no Brasil: uma (re) visão. Cien Saude Colet 2009; 14(1):297-305.

8. Kilsztajn S, Lopes ES, Lima LZ, Rocha PAF, Carmo MSN. Leitos hospitalares e reforma psiquiátrica no Brasil. Cad Saude Publica 2008; 24(10):2354-2362.

9. Mari JJ. Um balanço da reforma psiquiátrica brasileira. Cien Saude Colet 2011; 16(12):4593-4596.

10. Desviat M. Panorama internacional de la reforma psiquiátrica. Cien Saude Colet 2011; 16(12):4615-4622.

11. Perez F, coordinador. Dos décadas tras la reforma psiquiátrica. Madrid: AEN; 2006.

12. Kamis-Gould E, Snyder F, Hadley TR, Casey T. The Impact of Closing a State Psychiatric Hospital on the County Mental Health System and Its Clients. Psychiatr Serv 1999; 50(10):1297-1302.

13. Adreolli SB, Almeida-Filho N, Martin D, Mateus MDML, Mari JJ. É a reforma psiquiátrica uma estratégia para reduzir o orçamento da saúde mental? O caso do Brasil. Rev Bras Psiquiatr 2007; 29(1):43-46.

14. Dias MD, Araújo A, Amorim A, Salgado D, Costa M, Ferreira D. Corpo e desinstitucionalização em Saúde Mental: construindo práticas de reabilitação psicossocial. $3^{\circ}$ Congresso Nordeste de Ciências do Esporte; 2010; Fortaleza, CE [acessado 2013 fev 13]. Disponível em: www.rbceonline.org.br/congressos/index.php/conece/ 3conece/paper/viewFile/2466/996.

15. Morgan NC, Jonhson NA. Failures in psychiatry: the chronic hospital patient. AJP 1957; 113(9):824-830.

16. Barros S, Bichaff R, organizadores. Desafios para a Desinstitucionalização: Censo Psicossocial dos moradores em hospitais psiquiátricos do estado de São Paulo. São Paulo: FUNDAP; 2008.

17. Vieira TS, Monteiro CFS. Serviços residenciais terapêuticos: um dispositivo de reinserção social no contexto da reforma psiquiátrica. Rev NOVAFAPI 2010; 3(3):4448.

18. Alencar BL. Serviços de residências terapêuticas: entre a tutela e a autonomia, a incansável busca pelo cuidado... [dissertação]. Recife: Universidade Federal de Pernambuco; 2009.

19. Rosa MAS, Mutchnik VI. A Temporalidade no serviço de residência Terapêutica. $3^{\circ}$ Congresso Ibero-americano de Psicogerontologia; 2009; São Paulo, SP [acessado 2013 fev 13]. Disponível em: http://www.geracoes.org. br/arquivos_dados/foto_alta/arquivo_1_id-169.pdf.
20. Davidson M, Harvey PD, Powchik P, Parrella M, White L, Knobler HY, Losonczy MF, Keefe RS, Katz S, Frecska E. Severity of symptoms in chronically institutionalized geriatric schizophrenic patients. AJP 1995; 152(2):197-207

21. Harvey PD, Silverman JM, Mohs RC, Parrella M, White L, Powchik P, Davidson M, Davis KL. Cognitive decline in late-life schizophrenia: a longitudinal study of geriatric chronically hospitalized patients. Biol Psychiatry 1999; 45(1):32-40.

22. Lojudice, DC, Tonini NS, Santos SA, Schneider JF. Residências terapêuticas e reabilitação psicossocial: relato de experiência. $1^{\circ}$ Seminário Nacional de Estado e políticas sociais no Brasil; 2003; Cascavel, PR [acessado 2013 fev 13]. Disponível em: http://cac-php.unioeste. br/projetos/gpps/midia/seminariol/trabalhos/Saude/ eixo2/23nelsisalete.pdf

23. Harvey PD, Lombardi J, Leibman M, Parrella M, White L, Powchik P, Mohs RC, Davidson M, Davis KL. Age-Related Differences in Formal Thought Disorder in Chronically Hospitalized Schizophrenic Patients: A Cross-Sectional Study Across Nine Decades. AJP 1997; 154(2):205-210.

24. Burvill PW, Mittelman M. A follow-up study of chronic mental hospital patients 1959-1969. Social Psychiatry 1971; 6(4):167-171.

25. Duarte YAO, Andrade CL, Lebrão ML. O Índex de Katz na avaliação da funcionalidade dos idosos. Rev Esc Enf USP 2007; 41(2):317-325

26. Converso MER, Iartelli I. Caracterização e análise do estado mental e funcional de idosos institucionalizados em instituições públicas de longa permanência. J Bras Psiquiatr 2007; 56(4):267-272.

27. Luchins DJ, Goldman MB, Lieb M, Hanrahan P. Repetitive behaviors in chronically institutionalized schizophrenic patients. Schizophr Res 1992; 8(2):119-123.

28. Ribeiro IP. De volta para casa: estudo com egressos de hospitais psiquiátricos [dissertação]. São Bernardo: Universidade Metodista de São Paulo; 2009.

29. Katz S, Ford AB, Moskowitz RW, Jackson BA, Jaffe MW. Studies of Illness in the Aged: The Index of ADL: A Standardized Measure of Biological and Psychosocial Function. JAMA 1963; 185(12):914-919.

30. Chaves AC, Shirakawa I. Escala das síndromes positiva e negativa e seu uso no Brasil. Rev Psiquiatr Clín 1998; 25(6):337-343.

31. Bezerra CG. Moradores crônicos de hospital psiquiátrico: um desafio à desinstitucionalização [dissertação]. Natal: Universidade do Rio Grande do Norte; 2010.

32. Leff J, Trieman N. Long-stay patients discharged from psychiatric hospitals. BJP 2002; 176(3):217-223.

33. Brasil. Ministério da Saúde (MS). Datasus. Cadastro Nacional dos Estabelecimentos de Saúde [acessado 2013 fev 13]. Disponível em: http://cnes.datasus.gov. br/Index.asp?home=1.

34. Conceição MAT. O desafio da desinstitucionalização nos hospitais psiquiátricos. Jornal Cruzeiro do Sul. [acessado 2013 fev 13]. Disponível em: http://portal. cruzeirodosul.inf.br/acessarmateria.jsf?id=277882. 
35. Liberado MDM. Desinstitucionalizar é ultrapassar barreiras sanitárias: o desafio da intersetoriedade e do trabalho em rede. Cad Bras Saúde Mental (CD-ROM) 2009; 1(1) [acessado 2013 fev 13]. Disponível em: stat.saudeetransformacao.incubadora.ufsc.br/index. php/cbsm/article/view/1016/1142.

36. Oliveira MSN, Pinto FJM, Aguiar JB, Sampaio RMM, Medeiros CRBL. Perfil sociodemográfico e clínico de pacientes em internações psiquiátricas voluntárias e involuntárias. Rev Bras Promoç Saúde 2001; 24(4):361366.

37. Brasil. Ministério da Saúde (MS). Datasus. Estado Nutricional - Usuários da Atenção Básica - Ceará [acessado 2013 fev 13]. Disponível em: http://tabnet.datasus. gov.br/cgi/tabcgi.exe?sisvan/cnv/acom_CE.def

Artigo apresentado em 19/10/2013

Aprovado em 20/10/2013

Versão final apresentada em 21/10/2013 